\title{
Research on Developing Strategy in Xinjiang Uygur Medicine Tourism Industry
}

\author{
Xiaoqin Zhang ${ }^{1, \mathrm{a}}$ Zhaoyu Liao $^{2, \mathrm{~b}}$ \\ ${ }^{1}$ College of Economics and Business Administration, Tarim University, Xinjiang, China \\ ${ }^{2}$ Vice president of Tarim University Institute of western culture, Tarim University, Xinjiang, China \\ aemail, bemail,
}

Keywords: Uygur Medicine, Cultural Tourism Industry, Development Strategy

\begin{abstract}
Xinjiang is not only a multi-ethnic region, but also the main production areas of the Uygur Medicine, there is a very profound cultural heritage of medicine, with the developing of the cultural tourism about the advantages on Uygur medicine. Promoting the development of the cultural tourism industry of Uygur medicine can help improve the development of Xinjiang's economy a lot. This paper analyzes the advantages of the development of cultural tourism industry in Xinjiang Uygur Medicine, and provides a choice of strategy for developing tourism industry.To promote the whole xinjiang Uygur Medicine culture rapidly and provide reference for the tourism industry.
\end{abstract}

\section{Introduction}

Uygur Medicine, as an important part of intangible cultural heritage, still brought benefits to masses in Xinjiang, its efforts cannot be ignored. With the developing of the social economy, according to their own advantages and disadvantages, many enterprises have begun to reform to find suitable way of their own . Uygur Medicine is not only carrier of years history and culture, but also including strong ethnic and regional characteristics, the development is the trend of Uygur Medicine. Xinjiang is the main production area of Uygur Medicine, which has a profound cultural heritage of Chinese medicine, and it owns diversify cultural tourism resources . During developing period, we can not focus on old models, we should pay attention to its own advantages, and dig its tourism value, find different methods to this. If so, it will not only upgrades of Xinjiang's economy, but also bring more cultural products for consumers.

\section{The Significance in Developing the Cultural Tourism Industry of Uygur Medicine in Xinjiang}

To Promote Upgrading of Xinjiang's Economy Rapidly. Uygur Medicine is an integral part of Chinese traditional medicine, and it is also the major support of the medical service in Xinjiang. It plays an irreplaceable role among the economy and the health of our nation. The development of social diversification requires that Uygur Medicine should not be limited by the development model of traditional industries, but should keep up with time, seek new ways of development. To the medical knowledge and pharmacological knowledge, Medicine culture tourism is a carrier, to create economic effect by traveling, as traditional medicine culture is broad and profound, Uygur medicine culture tourism development can not only promote the development of tourism in Xinjiang, but also can drive health care industry, cosmetic industry and other related services developing, promote the whole economy the ascent in Xinjiang effectively.

Get Public to Understand Xinjiang Better. Since ancient times, Xinjiang had become a mixed place of variety cultures and owns longstanding history because of its geographical 
position.However, due to some negative events, many people have a narrow understanding of Xinjiang and forget its brilliant civilization gradually. Xinjiang is a magical place.people in Xinjiang are good at singing and dancing, there is a profound historical and cultural in Xinjiang. For thousands of years, according to the experience and on the basis of Traditional Chinese Medicine (TCM) ,Uygur people formatted and developed a complete system of medicine with national characteristics and regional characteristics .it is the wisdom of the whole nation, it saved thousands of lives countless times in history. Now, by traveling, it can be understood by more people, even the culture of Uygur Medicine, the Uygur ethnic, and Xinjiang.

Effective Protection and Inheritance of National Medicine. Uygur Medicine is not only an indispensable part of Traditional Chinese Medicine, but also a wealth of mankind. As a category of intangible cultural heritage, it is not only the epitome of the context of national history and traditional culture, but also maintaining the national sentiment of the cultural foundation and link of the most important health resource in Xinjiang contemporary. To benefit people, how it will continue to inherit and develop down, we must complete the mission. Practice to prove that the development is the best way to protect and inherit, the spread of tourism will undoubtedly widen the channel for the development of Uygur Medicine. This is not only the effective protection and heritage of the Uygur Medicine, but also build a big cultural province.

\section{The Advantages of Developing on Xinjiang Uygur Medicine Culture Tourism Industry}

Xinjiang Has A Profound Uygur Medicine Culture. Uygur Medicine is a native medicine.For more than two thousand years, Tarim Basin, northern and southern Uygur people, who live in North and south of the Tianshan Mountains, struggled with the disease, through constantly accumulation and sum up the experience of preventing and curing diseases.Uygur Medicine,under the influence of traditional Chinese medicine, combined with the national characteristics and environment, and slowly developed into a set of system medicine. Its efficacy has gradually been recognized by the entire medical community, but also loved by people of all ethnic groups. Uygur Medicine is a door, with a long history of ancient ethnic medicine, set obvious regional characteristics and national characteristics in a body. Ghazibay grew up in this piece of land ,who is the ancestor of the Uygur Medicine, Ghazibay medicine book, Happiness wisdom, Turkic Dictionary, and so on ,these medical works also come from this land.

The Belt and Road Has Injected New Vitality for It. In ancient times, with its superior geographical position,the western became a variety of cultural convergence, but on the ancient Silk Road . With the advance of "The Belt and Road" strategy, now Xinjiang has become the center area of the new Silk Road Economic Belt, is the national key supporting area. New Silk Road Economic Belt Construction will drive multiple transportation and modern logistics industry development, also promote capital flowing, technology, talent, and these conditions provide a powerful guarantee for the development of cultural tourism industry in Uygur Medicine.

Policy Provides A Strong Support for Its Development. In recent years, tourism industry and traditional medicine are highly valued by our country, the medical culture and tourism as a new mode of integration, governors takes much attention to healthy development. From 2014 to 2016, the State Council issued several documents on the tourism industry which put forward new requirements, related to infrastructure, marketing, environmental, regulatory and other aspects. It has stressed to find tourism value of culture of medicine, with their own advantages in resources, provides the treatment, health care, pension and integrated for visitors at the same time. And these documents carry the knowledge of medical culture to others. These policy documents guarantee for the development of Xinjiang Uygur Medicine culture tourism. 


\section{Developing Situation of the Cultural Tourism Industry of Xinjiang Uygur Medicine}

The Concept of Medical Cultural Tourism Has Not Yet Been Formed. Since ancient times, everyone thought that Uygur Medicine protect human from diseases and took care of the perfect combination. Even though the concepts of developing cultural tourism has not yet been formed, and medical culture of tourism destination become less and less. To the medical culture ,medicine culture tourism is a carrier, by the way of combining traveling treatment, health care, culture experience, provide consumers with more new tourism services, the combination of the pharmaceutical industry and the tourism market is good way of developing Uighur Medicine. So far, Xinjiang has no a mature route for medical culture tourist, products and project about tourism is little, too, degree of extensive public participation is also low.

Resource Advantages Have Not Yet Been Transferred into Industrial Advantages. Xinjiang Uygur Medicine culture resources are variety that any other provinces cannot achieve, as the medical knowledge and treatment methods in history are inherited by family , but it lack of systematic teaching, and due to different language, as a result, many medical data cannot collect or sort. They did not do a systemic developing in famous monuments, medical heritage and literature ,either.There are a lot of good physiotherapy and manipulation in Uygur Medicine, such as sand therapy, egg therapy, snow lotus dietotherapy, these therapies are simple,natural and quite efficacy, and be loved by people from all ethnic groups. but we did not make good use of these therapies and we did not discover it very well. In short, Xinjiang's medicine and cultural resources have not been fully transferred into tourism resources, we did not excavate its value .we should open our market.

Lack Professional Talent Resources. The development of enterprises and talent can not be separated, for the medical and cultural tourism is even more like this. Professional talent is the core of the Uygur Medicine culture tourism. Whether can we connect the Uygur Medicine culture and its spiritual value wealth that show every tourist is the most basic professional quality. And now both of them have medical knowledge, but they do not have the quality of tourism professionals. Talent as the core of the development of the enterprise, without it, it will be the biggest problem in enterprise development, and this will seriously hinder the development of national medicine culture tourism.

\section{Strategy in Developing Xinjiang Uygur Medicine Culture Tourism Industry}

Develop A Wealthy Medicine and Cultural Tourism Products. Uygur Medicine culture itself contains much content. It covers the knowledge of medicine and pharmacology, and relates to the national culture, diet habit, folk customs and so on. In addition to the profound historical and cultural, there is also many tourism resources. The diversify of tourism products will affect the number of visitors directly, it is essential that how to develop these intrinsic things into a considerable, can be rewarded, the purchase of tourism products to meet the needs of consumers. In order to develop medicine culture tourism for the long run, it is necessary to produce more medicine and cultural tourism products and carry out a variety of medical projects. Develop the experience of travel, health care, education, travel and so on. We should develop a series of tourism products, including Uygur medical appliances, a handbook of medical knowledge, health care products and souvenirs, etc, allowing visitors to be able to enjoy all kinds of Uygur Medicine tourism services at the same time, to buy tourism products that are good for their body.

Advertise More about Uygur Medicine Culture. As a matter of bad traffic and communication, Xinjiang and the mainland did not communicate very well, and this is the main reason that a lot of 
people know little about the culture and effect of Uygur Medicine. However, with the rapidly developing of the Internet, mobile broadband and other new round of communications gradually break the geographical restrictions, so that distance is no longer a problem any more. We should seize the existing conditions, make most use of television broadcasting, network media, periodicals, magazines and other media to advertise Uygur Medicine culture. Have a good knowledge of Uygur Medicine about excellent sand therapy culture, diet culture, health care culture, and to create a good brand of tourism culture. Make most use of the advantages of the Internet, to complete set of network marketing platform, through these different media and channels to enhance the reputation of the medical culture and tourism .

Go Abroad and Do Overseas Marketing. National culture is a part of world culture, Uygur Medicine although it is an important part of Chinese excellent traditional culture. It is also a precious heritage of all mankind as a medicinal resources, it should make effort in providing mankind health. It should not only be confined in Xinjiang, or in China, it should explore international market. Xinjiang is a variety of cultural exchange center because of geographical location in the past, and now this advantage is still existing. Xinjiang is one of the largest countries provinces which connect with other countries, no matter in language, religion, or ethnic customs and habits, it has common with neighboring countries , and this is good for Uygur Medicine culture developing. "The Belt and Road" strategy bring opportunity Uygur Medicine, for this, we should explore overseas market of Uygur Medicine.

Cultivate A Team of Outstanding Talents. At present, the main problem is to solve the problem of shortage in developing of Uygur Medicine culture tourism, whether you can find team with a strong professional knowledge, good service attitude, service level high personnel related to the enterprise's success or not. For vary the way of personnel training, both in Colleges and universities to meet the requirements of the relevant professional, we can also offer short-term training courses. For different positions, different languages, train people from different fields, and fully guarantee the quality of personnel and business level.Bring some new things to Uygur Medicine culture tourism.

\section{Acknowledgements}

Fund Project: National cultural science and technology innovation project: Tarim intangible cultural heritage tourism comprehensive service system construction and Application(number: 2013BAH27F00)

\section{References}

[1] Xiaowen Li, Qingping Xie. On the culture of Traditional Chinese Medicine industry [J]. Asia-Pacific Traditional Medicine. 2014,01:6-7.

[2] Xiaoping Chen, Xiangru Sun, Qinghu He. Study on the bottleneck and Countermeasures of the development of traditional Chinese medicine health preserving culture industry [J]. Journal of Hunan University of Chinese Medicine. 2014, 04:62-65.

[3] Haiyuan Peng, Xiangzhi Zhu, Fan Hong, Fangren Wang. Research on the current situation and Countermeasures of the development of Traditional Chinese Medicine culture industry in Fujian Province [J]. China Medical Herald.2014, 2 8:120-123.

[4] Yan Zhao. An analysis of the important role of the library of Xinjiang Medical University in promoting the brand and the development of the medical culture of Uygur Medicine [J]. Science \& Technology Information.2012,18:200+202. 
[5] Qian Wang, Weifan Yu. Heng Kong. Economic Thinking on building the brand industry of Uygur Medicine[J]. Market Modernization.2014,26:127-128.

[6] Xiaoguang Jia, Xiaojin Li. Xinjiang medicinal resources and development of Uygur Medicine industry [J]. Science \& Technology Industry Parks .2015,02:60-62.

[7] Yaodong Qi, Liping Li, Peigen Xiao. Uighur Medicine on the Silk Road [J]. Modern Chinese Medicine. 2016,03:375-378.

[8] Yang Yang, Ying Huang, Difan Zhang. An analysis of the development of Traditional Chinese Medicine cultural and creative industries with the characteristics of Yunnan [J]. Soft Science of Health.2015,10:605-608. 